\title{
EXPLAINING AI MODELS FOR CLINICAL RESEARCH: VALIDATION THROUGH MODEL COMPARISON AND DATA SIMULATION
}

\author{
Qing Zeng-Treitler ${ }^{1,2, a}$, Yijun Shao, 2, a, Douglas Redd ${ }^{1,2, \text { a }}$, Joseph Goulet ${ }^{3,4, \text { a }}$, Cynthia Brandt 3, 4, b \\ and Bruce Bray ${ }^{5, \mathrm{~b}}$ \\ ${ }^{1}$ George Washington University, 2121 I Street, NW, Washington, DC 20052, USA \\ ${ }^{2}$ DCVA Medical Center, 50 Irving St NW, Washington, DC 20422, USA \\ ${ }^{3}$ Yale University Medical School, 333 Cedar Street, New Haven CT 06510, USA \\ ${ }^{4}$ West Haven VA Medical Center, 950 Campbell Ave, West Haven, CT 06516, USA \\ ${ }^{5}$ University of Utah Medical School, 30 N. 1900 E, Salt Lake City, Utah 84132, USA \\ ${ }^{a} \mathrm{PhD}$ \\ ${ }^{b} M D$
}

\begin{abstract}
For clinical research to take advantage of artificial intelligence techniques such as the various types of deep neural networks, we need to be able to explain the deep neural network models to clinicians and researchers. While some explanation approaches have been developed, their validation and utilization are very limited. In this study, we evaluated a novel explainable artificial intelligence method called impact assessment by applying it to deep neural networks trained on real world and simulated data. Using real clinical data, the impact scores from deep neural networks were compared with odds ratios from logistic regression models. Using simulated data, the impact scores from deep neural networks were compared with the impact scores calculated based on the ground truth (i.e. formulas used to generate the simulated data). The correlations between impact scores and odds ratios ranged from 0.63 to 0.97 . The correlations between impact scores from DNN and ground truth ranged were all above 0.99. These suggest that the impact score provide a valid explanation of the contribution of a variable in a DNN model.
\end{abstract}

\section{KEYWORDS}

Explainable AI, Validation, Clinical Research

\section{INTRODUCTION}

The roots of artificial intelligence (AI), with the neural network as a signature technology, span many decades. Many of the algorithms, however, could not be productively implemented with the technology available in years past. Improvements in computing power have fueled renewed enthusiasm in AI, enabling implementation of algorithms that were previously strictly theoretical. In particular, deep learning methods have resulted in very good performance in speech recognition and many computer vision tasks. It also produced stunning results in authentic game play. Deep learning approaches have advantages over more traditional statistical and machine learning methods due to their ability to model highly non-linear relationships and the avoidance of the need for extensive feature engineering. With impressive results ranging from research to consumer products, efforts have been undertaken to generalize these approaches to widespread domains. This has resulted in some inevitable exaggerated claims and unfortunate false steps.(Quach 2019) There have also been many preliminary successes in important areas.(Rajkomar, Oren et al. 2018)

AI has shown promise for many clinical research applications.(Miotto, Wang et al. 2017) With the widespread adoption of electronic health record (EHR) systems, large amounts of clinical data have become available. This has the potential to fill the need of deep learning for large volumes of labeled data. Prediction of disease risk and hospital readmission are often challenging for traditional machine learning methods, and deep neural networks (DNNs) are beginning to have an impact.(Weng, Reps et al. 2017) For example, a 
DNN has been successfully used to identify diabetic retinopathy from retinal images.(Gulshan, Peng et al. 2016) Additionally, techniques such as recurrent neural networks (RNNs) are showing great promise for modeling time series data from EHRs.(Miotto, Li et al. 2016)

At the same time, deep neural network (DNN) models are viewed as black boxes, and understanding and explaining their behaviors is the subject of ongoing research. To influence clinical practice, a prediction model must have sufficient discriminating power, calibration, face validity and clinical utility. Clinicians are less inclined to act on predictions made by black-box models and researchers generally would like to investigate the significance of features (predictors) of a model. Thus, to gain clinical and research acceptance and impact, we need to provide interpretation of the DNN models.

Compared to clinical data, images of natural scenes, recordings of speech, or game board layouts are much easier for humans to understand. As a result, the majority of the DNN explanation work that has been published is intended to confirm human interpretation, e.g., if an image feature critical to the prediction of being a dog looks like the outline of a dog. With clinical prediction, human users do not have such prior knowledge and expect the model to yield information that justifies the outcome prediction.

Approaches that are being explored to explain DNN models include Attention Mechanism (Park, Hendricks et al. 2017), Local Interpretable Model-Agnostic Explanations (LIME)(Ribeiro, Singh et al. 2016) and Layer-wise Relevance Propagation (LRP).(Binder, Montavon et al. 2016) Each of these approaches has strengths and limitations: Attention mechanism provides insight into the DNN process, but can also be configured in different ways to alter the DNN process. LIME is agnostic to the DNN structure, though the manipulation of input is typically performed one variable at a time. LRP must make assumptions of the weight distribution within a neural network.

One area of ongoing research is to interpret and draw statistical inferences based on the explanations generated by these approaches.(Adadi and Berrada 2018, Kim, Rajaraman et al. 2019) Another is to validate the DNN explanations.(Lamy, Sekar et al. 2019) This paper focuses on the validation of a DNN explanation method, Impact Assessment (IA)(Shao, Cheng et al. 2019), which is developed by our team. Please note that DNN performance, though important, is not the focus of this paper.

\section{METHODS}

\subsection{Background}

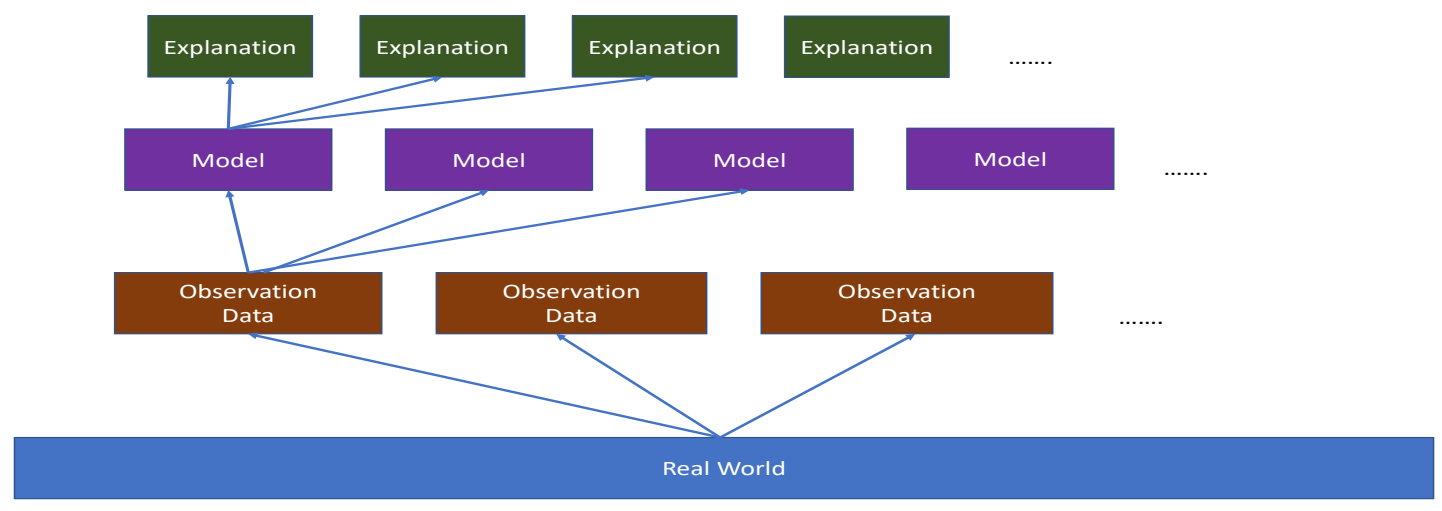

Figure 1. Relationships among real world use cases, data, model, and model explanation

Validation is an integral part of machine learning. As shown in Figure 1, multiple observation datasets may be available for one real world use case (e.g. re-hospitalization or mortality after surgery). Multiple models may be trained on a dataset and multiple explanations may be derived from a model. For the assessment of model performance and generalizability, internal and external validations are often carried out. Internal validation uses a subset of the same dataset to validate a model fitted on a different subset, while external 
validation uses a different dataset to validate a model fitted on a certain dataset. To understand a model's strengths and weaknesses, model comparison is also commonly performed. It typically evaluates one model using another as the reference or evaluates multiple models based on "ground truth", all on the same datasets. When ground truth is difficult to establish using data from the real world, however, validation through simulated data can be very helpful. AI models are frequently evaluated through internal and external validation. Explanation of AI models, however, cannot be easily validated against the ground truth because patterns, relationships, and pathways in the natural law that are only partially captured the observational data. In the study we describe in this paper, we compare explanations of different models trained on the same datasets. We also compare the explanation with ground truth in simulated data.

The Impact Assessment (IA)(Shao, Cheng et al. 2019) method we developed belongs to the LIME approach. For each variable, IA calculates a new measurement called impact score. Similar to the log-odds ratio from logistic regression, the Impact score represents the average rate of change of log-odds of the predicted risk.

The calculation begins by selecting a reference value. For binary variables such as diagnoses, 0 is selected as the reference as it indicates the absence of a diagnosis. For categorical variables (e.g. race), we select a category (e.g. unknown) based on convention and clinical context as the reference. For continuous variables, the mean is typically used as the reference. We then change a variable's current value to the reference value and observe change on the outcome. If the outcome does not change, this suggests that the change in value has no impact (as compared to the reference value). If the model changes, we will proceed to the following calculation.

The last layer of the DNN outputs a value $p$ between 0 and 1 though a sigmoid function $p=\sigma(x)$. The change of prediction originates from change in $x$. One way to obtain $x$ from $p$ is the logit function: $x=\operatorname{logit}(p)=\log \frac{p}{1-p}$ Therefore, the individual-level impact score is defined as:

$$
\frac{\operatorname{logit}\left(p_{\text {cur }}\right)-\operatorname{logit}\left(p_{\text {ref }}\right)}{\text { (current value })-(\text { reference value })}
$$

where: $p_{\text {ref }}$ is the new value of $p$ after changing the current value to reference value.

Each individual's impact score on a given variable varies because the DNN model can model non-linear relationships between the predictor and outcome variables. We define the impact score at the population level simply as the mean of individual scores.

\subsection{Observation Data}

We used data from two different clinical research studies: 1) Frailty - this study predicted the outcome of patients following major cardiovascular procedures using patient demographics and clinical characteristics including frailty status which is extracted from clinical notes; and 2) Complementary Integrative Health $(\mathrm{CIH})$ - this study attempts to identify the clinical and demographic features associated with CIH use in patients with muscular skeletal disorder.

The number of features and sample size of each study is shown in Table 1.

Table 1. Characteristics of the real world datasets

\begin{tabular}{|c|c|l|c|c|}
\hline & Outcome & \multicolumn{1}{|c|}{ Features } & $\begin{array}{c}\text { Number of } \\
\text { Features }\end{array}$ & Sample Size \\
\hline Frailty & Mortality & $\begin{array}{l}\text { Demographi } \\
\text { cs, comorbidities, } \\
\text { prior } \\
\text { hospitalization, } \\
\text { prior diagnoses, } \\
\text { vital signs, frailty } \\
\text { status }\end{array}$ & 57 & 21,355 \\
\hline CIH & $\begin{array}{l}\text { Usage of } \\
\text { Acupuncture, } \\
\text { Biofeedback, } \\
\text { Guided Imagery, } \\
\text { Meditation, Tai- } \\
\text { Chi, or Yoga }\end{array}$ & $\begin{array}{l}\text { vital signs, Opioid } \\
\text { use }\end{array}$ & 19 & \\
\hline
\end{tabular}




\subsection{Validation through Model Comparison}

Different DNN models were fitted to the 2 datasets (Table 2), while 2 logistic regression models were also fitted to each of the same datasets. IA was applied to the DNN models, with each variable's impact score as the explanation. The DNN structure was determined empirically in each case. For logistic regression, we used the odds ratio of each variable as the explanation. The Pearson and Spearman correlation was calculated between the impact scores and odds ratios for each dataset.

Table 2. DNN model description and DNN/logistic regression model performance

\begin{tabular}{|c|l|c|c|}
\hline railty & \multicolumn{1}{|c|}{ DNN Model Description } & $\begin{array}{c}\text { DNN } \\
\text { Performance } \\
\text { F }\end{array}$ & $\begin{array}{l}\text { Togistic } \\
\text { branch taking temporal data (represented as an image) as } \\
\text { input was a convolutional neural network with 5 hidden } \\
\text { performance }\end{array}$ \\
\hline $\begin{array}{l}\text { layers. The branch taking non-temporal data has one fully- } \\
\text { connected layer. The last layers from the two branches were } \\
\text { joined using a simple concatenation to form the last hidden } \\
\text { layer of the whole network. The initial weights in all the } \\
\text { hidden layers and the output layer of the DNN were } \\
\text { randomly assigned with small numbers. Then the DNN were } \\
\text { trained on the training set with weights updated iteratively } \\
\text { using back propagation. }\end{array}$ & AUC=0.787 & AUC=0.746 \\
\hline IH & $\begin{array}{l}\text { The DNN was constructed with an input layer of 19 } \\
\text { nodes, corresponding to the 19 variables, and an output layer } \\
\text { of a single node giving the probability of CIH use. Five fully } \\
\text { connected hidden layers were used, all using the rectified } \\
\text { linear unit function for activation. Weights were initialized } \\
\text { with random small numbers and updated using stochastic } \\
\text { gradient descent with Nesterov momentum. }\end{array}$ & AUC=0.683 & AUC=0.681 \\
\hline
\end{tabular}

\subsection{Simulated Data}

Two sets of simulation data are generated to allow us validate the explanations against the ground truth. We selected binary outcome because many clinical risk predictions tasks have binary outcomes or categorical outcomes that can be translated into multiple binary outcomes (e.g. mortality, hospitalization, incident diagnosis and adverse event). The number of predictors in clinical studies can vary from below 50 to over 1000 and we chose $n=100$ for this study. The inclusion of both linear relationship and non-linear relationship is driven by the desire to represent both simple and complex datasets.

First, we use 100 variables $x_{1}, x_{2}, \ldots, x_{100}$ as predictors and a binary variable $z$ for two outcomes represented by values 0 and 1 respectively. Among the 100 variables the first 50 are binary variables taking values $0 / 1$, and the second 50 are continuous variables taking values ranged between 0 and 1 . This setting is to resemble the real situation of patient data: some variables such as gender, diagnoses, procedures are usually treated as binary variables, while the other variables such as age, vital signs, lab results are usually treated as continuous variables. Although the continuous variables may have different numerical value ranges in their original form, they can always be normalized to range from 0 to 1.

We will experiment with two different types of relationship between the outcome variable $z$, or more specifically, the $\log$ odds $y=\log \frac{p}{1-p}$, where $p=\operatorname{Prob}(z=1)$, and the predictors: 1) linear relationship, and 2) non-linear relationship.

1) the linear relationship is given by

$$
y=\beta_{0}+\beta_{1} x_{1}+\cdots+\beta_{100} x_{100}
$$

The coefficients $\beta_{1}, \ldots, \beta_{100}$ are randomly generated from a uniform distribution with range -1 to 1 . Once the coefficients are generated, they are fixed for all the following analysis. We set $\beta_{0}=1$.

Next, we generate 50,000 vectors $\left(x_{1}, x_{2}, \ldots, x_{100}, z\right)$ also through a random process. The values of the first 50 variables are sampled from a Bernoulli distribution with $p=0.3$ (i.e., taking value 1 with probability 
$0.3)$. The values of the second 50 variables are sampled from a uniform distribution with range 0 to 1 . The values of the outcome variables $z$ is generated randomly from a Bernoulli distribution with probability $p$ calculated using the sigmoid function

$$
p=\frac{1}{1+e^{-y}}
$$

2) the nonlinear relationship is given $b$

$$
y=\beta_{0}+\sum_{i=1}^{100} \beta_{i} x_{i}+\sum_{i \in I} \gamma_{i} x_{i}^{2}+\sum_{j \in J, k \in K} \theta_{j k} x_{j} x_{k}
$$

where $I, J$ and $K$ are random subsets of the index set $\{1,2, \ldots, 100\}$ of size 20 such that $J \cap K=\emptyset$. So this relationship is added with 20 square terms and 20 product terms. The coefficients $\beta_{i}$ are generated in the same way as in 1). The coefficients $\gamma_{i}$ and $\theta_{j k}$ are generated in the same way as $\beta_{i}$.

Next we generate 50,000 vectors $\left(x_{1}, x_{2}, \ldots, x_{100}, z\right)$ in exactly the same way as 1$)$ except that the non-linear relationship given in 2) is used.

\subsection{Validation through Comparison with Ground Truth}

After the simulation data are generated, we train a deep neural network (DNN) model on the data. The DNN has an architecture as follows. It has an input layer of 100 nodes and an output layer with 1 node. There are 5 hidden fully-connected layers between the input layer and output layer. Their numbers of nodes, from the first to the last, are 50,70,50,70 and 50, respectively. Their nonlinear activation functions are all the rectified linear unit function $f(x)=\max (0, x)$. The nonlinear activation function for the output layer is the sigmoid function $\sigma(x)=1 /\left(1+e^{-x}\right)$ so that the DNN outputs a number between 0 and 1 representing the predicted probability, $\operatorname{Prob}(z=1)$. This DNN architecture is empirically designed. Since our focus is on the explanation, we did not experiment with different architectures.

For each of the two simulated datasets (linear and non-linear), we divide the dataset into 3 random subsets: training (60\%), validation $(20 \%)$ and testing $(20 \%)$, and then we train the DNN model on the training set. The weights of the DNN is initialized with randomly generated small numbers before training, and updated using the Nesterov algorithm with a mini-batch size of 100, a learning rate of 0.001 and a momentum of 0.9 during training. After each epoch of training, the trained DNN model is tested on the validation set to measure the AUC. When the AUC reaches a peak point such that it shows no improvement over the following 10 epochs, we take the DNN model with the peak AUC as the final model.

With the final DNN model, we calculate impact scores as follows. Let $p=F\left(x_{1}, \ldots, x_{100}\right)$ be the final DNN model. By the design of DNN, we have $0<p<1$. First, we choose a reference value for each variable, which is zero for all variables in this case. We then proceeded to calculate the impact score as described in the Background section.

To verify the population-level impact scores calculated based on the learned DNN model, we need to calculate the true population-level impact scores based on the true underlying relationship used to generate the dataset. The calculation is similar as above, except that for the individual-level impact score formula, $\operatorname{logit}\left(p_{c u r}\right)$ and $\operatorname{logit}\left(p_{\text {ref }}\right)$ are replaced by $y_{\text {cur }}$ and $y_{\text {ref }}$, respectively, which are the $y$ defined in the true relationship with $x_{i}$ taking the current value/reference value. This change in definition is because when generating the data, $y$ is calculated ahead of $p$ using the sigmoid function.

We used several metrics to measure the difference between the true impact scores and the learned impact scores (by the DNN models), including mean absolute error (MAE), Pearson's correlation, and Spearman's (rank) correlation.

\section{RESULTS}

The comparison of impact score and odds ratio logistic regression using real world observation data show moderate to very strong correlations. (Table 3) This is consistent with the fact that the DNN models and logistic regressions models were trained on the same datasets. The DNN and logistic regression models are not expected to be the same, however, they are expected to have some similarities. 
Table 3. Correlation coefficients between impact scores and odds ratios

\begin{tabular}{|l|l|l|}
\hline & Pearson & Spearman \\
\hline Frailty & 0.69 & 0.63 \\
\hline CIH & 0.84 & 0.97 \\
\hline
\end{tabular}

Simulated evaluation results (Table 4) suggest that the DNN-derived impact score is highly correlated (Pearson's and Spearman's correlation coefficients > 0.99) with the impact score calculated based on the ground truth (i.e. the formulas used to generate the simulated score). The mean absolute errors are very small (<.07), given that the mean impact scores are around 0.5. An interesting observation is that the DNN achieved slightly higher AUC on the non-linear data and the correlations and absolute errors of impact scores are almost identical on the linear and non-linear data. Please note that when we compared the impact scores and odds ratios, the calculation of absolute errors was not possible as neither can be viewed as ground truth.

Table 4. Correlation coefficients and other performance metrics of impact score based on simulated data

\begin{tabular}{|l|l|l|}
\hline & Linear & Non-linear \\
\hline Training DNN Model & & 30 \\
\hline Number of Epochs & 30 & 0.891 \\
\hline Testing AUC & 0.873 & \\
\hline Impact Scores & & 0.065 \\
\hline Mean Absolute Error (MAE) & 0.058 & 0.514 \\
\hline Mean Absolute Predicted Value (MAPV) & 0.466 & 0.533 \\
\hline Mean Absolute Actual Value (MAAV) & 0.515 & $13 \%$ \\
\hline MAE / MAPV & $12 \%$ & $12 \%$ \\
\hline MAE / MAAV & $11 \%$ & 0.993 \\
\hline Pearson's Correlation & 0.997 & 0.990 \\
\hline Spearman's Correlation & 0.996 & \\
\hline
\end{tabular}

\section{DISCUSSION}

\subsection{Significances}

DNN models are a key AI approach. Recent studies have shown that DNN can outperform traditional machine learning (ML) methods and statistical models in clinical and non-clinical domains(Chen and Lin 2014, Belle, Thiagarajan et al. 2015, LeCun, Bengio et al. 2015, Tan, Gao et al. 2015, Pastur-Romay, Cedron et al. 2016, Erickson, Korfiatis et al. 2017, Lee, Jun et al. 2017, Miotto, Wang et al. 2017). This paper presents two approaches to validate a new explainable AI Method: model comparison and simulation. Results from both approaches suggest that the impact score provides a sensible explanation of the patterns captured by the AI models, and can be used to explain the real-world relationships. As AI is increasingly used by biomedical researchers, the demand for explanation is expected to grow. Therefore, the use of a method like IA is expected to increase as well.

\subsection{Implications}

Observational data is an imperfect reflection of the real world and often driven by specific clinical hypotheses and administrative requirements. Even clinical trial data have many flaws, e.g. there is often selection bias and there are always unobserved variables (which is assumed to be taken care of by the randomization process, but it is not guaranteed). Cohort studies, EHRs, insurance claims, and patient-reported datasets tend to be much larger and can have a very long follow-up, however, they also tend to be noisier and more complex to analyze.

Models are imperfect reflections of the data. As George Box said, "all models are wrong, but some are useful." Comparing models developed using real world data reflects the complexity in the real world. Different models may each represent the underlying ground truth partially, and thus offer different insights 
into the underlying ground truth. When we do not know the ground truth in real world data (e.g. we do not have the complete knowledge about the biological relationship between frailty and mortality), simulation provides us with ground truths. However, it builds on pre-defined rules and assumptions.

\subsection{Limitations}

Complex models can also be explained in different ways. There are already several approaches to explain deep learning models: some are based on an analysis of the inner structure and weights, while others are based on the change in outcome when the input is manipulated.

Currently, there is no ideal method to evaluate explanations of DNN. Model comparisons and simulations each have their strengths and weaknesses: statistical models cannot be viewed as a gold standard and simulated data tend to be simpler than real world observations. In addition, this study is limited by the model we chose for comparison, i.e. logistic regression. We chose logistic regression because the odds ratio yielded by this analysis is widely accepted by clinicians. There are of course many other modeling methods which can be compared with. There are also infinite ways to generate simulated datasets, and our simulated data can be made more complex.

\subsection{Future Work}

Clinical adoption requires explanations like impact scores. Our next step will be to test if impact scores can influence clinical decision making, i.e. the usefulness of impact scores. Our initial attempt at this revealed a number of challenges: e.g. when there are many variables, should we just show the ones with the highest impact? Should we display population and individual impact scores together? For continuous variables, should we provide information on change per unit or just the change? We will also need to go beyond the impact of single variables and explore multi-variable patterns.

\section{CONCLUSION}

We evaluated a novel explainable AI approach in two ways: comparison with the odds ratio from logistic regression and comparison with ground truth from simulated data. The results suggest the impact scores provide valid explanations of the contribution/importance of a feature in a DNN model. The impact scores can help facilitate the utilization of DNN in clinical research and practice.

\section{ACKNOWLEDGEMENT}

The work is funded in part by several US NIH and VA grants: 1UL1TR001876-01, I01 HX001145-02, 1I01HX002308-01A1, and R56 AG052536-01A1.

\section{REFERENCES}

Adadi, A. and M. Berrada (2018). "Peeking Inside the Black-Box: A Survey on Explainable Artificial Intelligence (XAI)." IEEE Access 6.

Belle, A., R. Thiagarajan, S. M. Soroushmehr, F. Navidi, D. A. Beard and K. Najarian (2015). "Big Data Analytics in Healthcare." Biomed Res Int 2015: 370194.

Binder, A., G. Montavon, S. Lapuschkin, K.-R. Müller and W. Samek (2016). Layer-wise relevance propagation for neural networks with local renormalization layers. International Conference on Artificial Neural Networks, Springer.

Chen, X. W. and X. T. Lin (2014). "Big Data Deep Learning: Challenges and Perspectives." Ieee Access 2: 514-525.

Erickson, B. J., P. Korfiatis, Z. Akkus and T. L. Kline (2017). "Machine Learning for Medical Imaging." Radiographics 37(2): 505-515. 
Gulshan, V., L. Peng, M. Coram, M. C. Stumpe, D. Wu, A. Narayanaswamy, S. Venugopalan, K. Widner, T. Madams, J. Cuadros, R. Kim, R. Raman, P. C. Nelson, J. L. Mega and D. R. Webster (2016). "Development and Validation of a Deep Learning Algorithm for Detection of Diabetic Retinopathy in Retinal Fundus Photographs." JAMA 316(22): 2402-2410.

Kim, I., S. Rajaraman and A. S (2019). "Visual Interpretation of Convolutional Neural Network Predictions in Classifying Medical Image Modalities." Diagnostics 9(2): 38.

Lamy, J., B. Sekar, G. Guezennec, J. Bouaud and S. B (2019). "Explainable artificial intelligence for breast cancer: A visual case-based reasoning approach." Artif Intell Med(94): 42-53.

LeCun, Y., Y. Bengio and G. Hinton (2015). "Deep learning." Nature 521(7553): 436-444.

Lee, J. G., S. Jun, Y. W. Cho, H. Lee, G. B. Kim, J. B. Seo and N. Kim (2017). "Deep Learning in Medical Imaging: General Overview." Korean J Radiol 18(4): 570-584.

Miotto, R., L. Li, B. A. Kidd and J. T. Dudley (2016). "Deep patient: an unsupervised representation to predict the future of patients from the electronic health records." Scientific reports 6: 26094.

Miotto, R., F. Wang, S. Wang, X. Jiang and J. T. Dudley (2017). "Deep learning for healthcare: review, opportunities and challenges." Brief Bioinform.

Miotto, R., F. Wang, S. Wang, X. Jiang and J. T. Dudley (2017). "Deep learning for healthcare: review, opportunities and challenges." Briefings in Bioinformatics 19(6): 1236-1246.

Park, D. H., L. A. Hendricks, Z. Akata, A. Rohrbach, B. Schiele, T. Darrell and M. Rohrbach (2017). Attentive Explanations: Justifying Decisions and Pointing to the Evidence. Interpretable ML Symposium (NIPS 2017). Long Beach, CA, USA.

Pastur-Romay, L. A., F. Cedron, A. Pazos and A. B. Porto-Pazos (2016). "Deep Artificial Neural Networks and Neuromorphic Chips for Big Data Analysis: Pharmaceutical and Bioinformatics Applications." Int J Mol Sci 17(8).

Quach, K. (2019, 18 Apr 2019). "IBM Watson Health cuts back Drug Discovery 'artificial intelligence' after lackluster sales." Retrieved 3 May 2019, from https://www.theregister.co.uk/2019/04/18/ibm_watson_health.

Rajkomar, A., E. Oren, K. Chen, A. M. Dai, N. Hajaj, M. Hardt, P. J. Liu, X. Liu, J. Marcus, M. Sun, P. Sundberg, H. Yee, K. Zhang, Y. Zhang, G. Flores, G. E. Duggan, J. Irvine, Q. Le, K. Litsch, A. Mossin, J. Tansuwan, D. Wang, J. Wexler, J. Wilson, D. Ludwig, S. L. Volchenboum, K. Chou, M. Pearson, S. Madabushi, N. H. Shah, A. J. Butte, M. D. Howell, C. Cui, G. S. Corrado and J. Dean (2018). "Scalable and accurate deep learning with electronic health records." npj Digital Medicine 1(1): 18.

Ribeiro, M. T., S. Singh and C. Guestrin (2016). Why should i trust you?: Explaining the predictions of any classifier. Proceedings of the 22nd ACM SIGKDD international conference on knowledge discovery and data mining, ACM.

Shao, Y., Y. Cheng, R. Shah, C. Weir, B. Bray and Q. Zeng-Treitler (2019). Shedding Light on the Black Box: Explaining Deep Neural Network Prediction of Clinical Outcome. ICHI 2019 : International Conference on Health Informatics. Rome, Italy.

Tan, S. S., G. Gao and S. Koch (2015). "Big Data and Analytics in Healthcare." Methods Inf Med 54(6): 546-547.

Weng, S. F., J. Reps, J. Kai, J. M. Garibaldi and N. Qureshi (2017). "Can machine-learning improve cardiovascular risk prediction using routine clinical data?" PloS one 12(4): e0174944. 